\title{
Reputation, Wahrheit und Blind Peer Review
}

\author{
Eine systemtheoretische Perspektive auf anonymisierte Autor- \\ schaft als Qualitätssicherungsstandard der Wissenschaften
}

\begin{abstract}
Dieser Beitrag setzt sich mit der Rolle von wissenschaftlicher Autorschaft in Verfahren zur Qualitätssicherung auseinander, wie sie im gegenwärtigen wissenschaftlichen Betrieb praktiziert werden. Peer Review ist dabei eine maßgebliche Praxis zur Bewertung von wissenschaftlichen Leistungen. Für akademische Zeitschriften ist die Beurteilung von eingereichten Manuskripten durch externe Gutachter zum Ausweis von Qualität avanciert. Aus verschiedenen Gründen wird dabei häufig die Identität der Autorinnen maskiert. Diese Praxis des Blind Peer Review ist in empirischen Studien auf seine Effektivität hin geprüft worden, die Ergebnisse bleiben bislang aber widersprüchlich. Eine theoretische Modellierung des Prozesses der Anonymisierung von wissenschaftlicher Autorschaft im Sinne der Systemtheorie Niklas Luhmanns zeigt, dass es strukturelle Aspekte des Blind Peer Review gibt, die in bisherigen Studien unberücksichtigt geblieben sind: Die Idee der Ent-Subjektivierung von Wahrheit und Wissen ist gekoppelt an bestimmte Prämissen, unter anderem an das Konzept des universalen Wissens. Dieses Konzept steht im Widerspruch zu einigen grundsätzlichen epistemologischen Annahmen, die in der Wissenschaft, und besonders in den Geisteswissenschaften, weit verbreitet sind.
\end{abstract}

\section{Wissenschaftliche Autorschaft und Qualitätssicherung}

Folgt man den Ausführungen Michel Foucaults zur Bedeutung von Autorschaft im gegenwärtigen Wissenschaftssystem, dann stößt man auf zwei Befunde. Einerseits, so Foucault, sei die Funktion Autor in der Wissenschaft »verwischt«, die »Wahrheit« würde durch die »Zughörigkeit zu einem systematischen Ganzen « abgesichert, nicht durch den Rückverweis auf eine bestimmte Person. Gleichzeitig, so Foucault weiter, sei aber in bestimmten Disziplinen die Namensnennung »ein `Glaubwürdigkeits «-Indiz «. ${ }^{1}$ Die Rezeption der Forschungs-

1 Foucault: Was ist ein Autor?, S. 12-13. 
ergebnisse hängt hier somit von dem Namen des wissenschaftlichen Autors ab. Unabhängig davon, ob man Foucaults Ausführungen als historisch angemessene Rekonstruktion der Entwicklung der Autor-Funktion versteht, ist diese doppelte Belegung des Autornamens in der Wissenschaft eine auch heute noch feststellbare Tatsache im Alltag wissenschaftlichen Handelns. ${ }^{2}$ Einerseits ist die Nennung von Eigennamen im Zusammenhang mit Forschungsergebnissen gängige Praxis: Wissenschaftliche Schriften werden mit Verweis auf den Namen ihrer Autorinnen zitiert und Literaturverzeichnisse in der Regel ebenfalls nach Namen sortiert. Auf der anderen Seite gibt es einen Teilbereich der wissenschaftlichen Praxis, in dem gerade nicht die Nennung des Autornamens, sondern der Verzicht darauf die Glaubwürdigkeit der Handelnden sicherstellen soll. Beim Blind Peer Review werden wissenschaftliche Forschungsergebnisse beurteilt, ohne dass den Wertenden der Name der Autorin des in Frage stehenden Textes bekannt ist. Bevor ich diese Praxis mit Hilfe einer systemtheoretischen Modellierung analysieren werde, möchte ich im Folgenden zunächst das Phänomen selbst skizzieren und auf einige bestehende Forschungen zum Thema Peer Review und Blind Peer Review hinweisen. Dies geschieht auch in der Annahme, dass die Praxis des Peer Review im Allgemeinen und des Blind Peer Review im Speziellen für die meisten Wissenschaftlerinnen zwar eine Selbstverständlichkeit darstellt, die wenigsten sich aber tatsächlich mit dem Für und Wider dieser gängigen Praktiken auseinandergesetzt haben und seine wissenschaftsgeschichtliche Entwicklung wenig bekannt ist.

\subsection{Peer Review}

Peer Review bedeutet die Evaluation von Wissenschaft durch Wissenschaftler und hat sich als zentraler Bestandteil der gegenwärtigen akademischen Praxis etabliert. Bei der Vergabe von Fördergeldern für Projektfinanzierungen oder Stipendien wird das Prinzip des Peer Review ebenso eingesetzt wie bei der Entscheidung für oder gegen die Aufnahme eines Textes in ein Verlags- oder Zeitschriftenprogramm. Wenn für die Vergabe einer Leistung, sei es die Publikation in einer Fachzeitschrift oder die Finanzierung eines Forschungsprojekts, die Meinung von Kollegen hinzugezogen wird, spricht man von Peer Review. ${ }^{3}$ In diesem Sinne ist Peer Review der zentrale Evaluationsmechanismus von Wissenschaft im aktuellen akademischen System.

2 Zur kritischen Diskussion von Foucaults Thesen vgl. Chartier: Foucault's Chiasmus.

3 Es hat sich hier auch im Deutschen der englischsprachige Begriff durchgesetzt. 
Im Folgenden soll ein spezieller Fall von Peer Review im Mittelpunkt stehen, und zwar Peer Review im Kontext von wissenschaftlichen Zeitschriftenpublikationen. Hier bedeutet Peer Review, dass ein wissenschaftlicher Beitrag vor seiner Veröffentlichung von Fachkollegen begutachtet wird, die dann über dessen Publikationswürdigkeit entscheiden. ${ }^{4}$ In dieser Form ist Peer Review mindestens seit dem 18. Jahrhundert bekannt. Je nachdem, welche Traditionslinien man als relevant ansieht, kann die genaue Datierung unterschiedlich erfolgen. John C. Burnham nennt als Ursprungsdatum das Jahr 1752, als die Royal Society of London für ihre Zeitschrift Philosophical Transactions ein >Committee on Papers einführt. ${ }^{5}$ Mario Biagioli verlegt die Ursprünge des heutigen Peer Review bereits in das 17. Jahrhundert. ${ }^{6}$

Als weit verbreitete, etablierte Praxis lässt sich Peer Review aber erst seit Mitte des 20. Jahrhunderts beobachten. ${ }^{7}$ In dieser Form des Peer Review sind mit den Peers in der Regel Fachkollegen gemeint, die nicht gleichzeitig Herausgeber der entsprechenden Fachzeitschrift sind: Peer Review meint also heute meist externe Gutachter. ${ }^{8}$ Erst nach dem zweiten Weltkrieg nutzen wissenschaftliche Zeitschriften auf breiter Basis regelmäßig solche Gutachter, um über die Qualität zu publizierender Artikel zu entscheiden. ${ }^{9}$

Diese Praxis ist zwar mittlerweile weit verbreitet, wird aber aus verschiedenen Gründen kritisiert. Der wichtigste Kritikpunkt lautet: Es ist nicht bewiesen -

4 Auch die Bewertung eines Beitrags durch Fachkollegen nach der Veröffentlichung kann mit dem Terminus »Peer Review« bezeichnet werden, liegt aber hier nicht im Zentrum des Interesses. Zum damit verbundenen Stichwort des Open Review s. Fitzpatrick: Peer-to-peer Review.

5 Burnham: Journal Editors, S. 56.

6 Biagioli: Censorship, S. 14. Biagioli nimmt mit Bezug auf Michel Foucault eine radikale historische Positionierung des Peer Review vor, wenn er die Verbindung des Imprimatur der Académie des Sciences und der Royal Society of London mit staatlichen Zensurpraktiken hervorhebt: „Still, it may not be out of place to view the long-term trajectory of the disciplining of printed texts as moving from early modern book-burning (the public material destruction of the text as object) to modern peer review (the internal disciplining of a text and its author) « (ebd., S. 11). Vgl. zur Verbindung von Peer Review und Zensur auch Casadevall / Fang: Censorship. Die Traditionslinien können noch weiter zurückgeführt werden. Ray Spier datiert "[p]erhaps the first documented description of a peer-review process « auf das 9. Jahrhundert (Spier: History, S. 357). Sein sehr weiter Begriff von `Peer macht aber eine Vergleichbarkeit eigentlich unmöglich, wie er selbst zugesteht.

7 Vgl. Burnham: Journal Editors, S. 55 und Haack: Peer Review, S. 794.

8 So auch die Definition von Ann Weller: "A peer-reviewed journal is one that has a portion of submitted manuscripts evaluated by someone other than the editor of the journal " (Weller: Editorial Peer Review, S. 16).

9 Vgl. für die historische Entwicklung u. a. Weller: Editorial Peer Review; Burnham: Journal Editors; Fitzpatrick: Peer-to-peer Review. 
und womöglich nicht beweisbar - dass die Wirkung des Peer Review den Aufwand lohnt. Eine Meta-Studie aus dem Jahr 2002 kommt zu dem Schluss: »Editorial peer review, although widely used, is largely untested and its effects are uncertain ${ }^{10}{ }^{10}$ Eine weitere Studie aus dem gleichen Jahr benennt ein wichtiges Problem in der Untersuchung von Peer Review: Die Ziele des Peer Review sind nicht eindeutig formuliert, weswegen es methodisch problematisch ist, seine Effektivität zu messen. ${ }^{11}$

Historisch gesehen, ist Peer Review ursprünglich als quantitativer Filter etabliert worden, um eine Vorauswahl wissenschaftlicher Arbeiten zu treffen. Susan Haack weist darauf hin, dass Peer Review aber heute häufig als positiver Indikator von Qualität verstanden wird. Während der Prozess ursprünglich nur sicherstellen sollte, dass minimale Standards erfüllt werden, verstehen manche das Label peer reviewed heute als Ausweis der Richtigkeit und Qualität eines wissenschaftlichen Beitrags. Peer Review, so Susan Haack, wird in seinen Funktionen überschätzt: »[A]s pre-publication peer review has spread and become entrenched in academic publishing and in the academy itself, some are tempted to exaggerate its virtues - to think of the system, not just as a rough-and-ready preliminary filter, but as a strong indication of quality «. ${ }^{12}$

$\mathrm{Zu}$ einem ähnlichen Schluss kommt Kathleen Fitzpatrick wenn sie feststellt, dass der Ausweis peer reviewed zum Beispiel in Berufungskommissionen häufig als Qualitätsindikator verstanden wird, um die tatsächliche Lektüre der Schriften der Kandidaten einzusparen. ${ }^{13}$ Eine Untersuchung der Modern Language Association of America kommt zu einem ähnlichen Schluss. ${ }^{14}$

Aber trotzdem scheint Peer Review ein alternativloses Prozedere zu sein, von dem große Teile der scientific community überzeugt sind. Herausgeber und Verlage halten an dem Prinzip des Peer Review fest. Richard Smith, ehemaliger Herausgeber des British Medical Journal bemerkt zu diesem Umstand: »How odd that science should be rooted in belief $«{ }^{15}$

10 Jefferson / Alderson u. a.: Effects, S. 2784.

11 Vgl. Jefferson / Wagner u. a.: Quality, S. 2786.

12 Haack: Peer Review, S. 796.

13 Fitzpatrick: Peer-to-Peer Review, S. 170.

14 Die Kommission der MLA stellt in Bezug zu Peer Review in Universitätsverlagen fest: »this apparatus of external peer review also created the conditions whereby individual departments can practically abdicate their responsibility to review the scholarly work of the very colleagues they have appointed to tenure-track positions« (Modern Language Association of America: Report, S. 56).

15 Smith: Peer review, S. 182. 
Dieser Mangel an verlässlichen Forschungsarbeiten zu Peer Review betrifft alle Disziplinen. ${ }^{16}$ In Bezug auf die Geisteswissenschaften führt Fitzpatrick zwei mögliche Begründungen für diese Forschungslücke an. Zum einen vermutet sie, dass es eine Art Angst vor den Ergebnissen einer solchen Untersuchung geben könnte. Die geisteswissenschaftliche Tradition, so Fitzpatrick unter Berufung auf David Shatz ${ }^{17}$ und Donald Hall, ${ }^{18}$ müsste in der Rückschau maßgeblich reevaluiert werden, wenn sich herausstellen würde, dass Peer Review im momentan praktizierten Sinne nicht optimal sei. Es gäbe in den humanities aber konservative Impulse, die eine solche Neubewertung etablierter Praktiken verhindern möchten. ${ }^{19}$ Als zweite Begründung, warum Peer Review nicht der kritischen Untersuchung unterzogen wird, mit der Geisteswissenschaftler sich üblicherweise ihren Gegenständen widmen, führt Fitzpatrick ein spezifisches Verständnis von >Wahrheit` an, das die Geisteswissenschaften von den sciences unterscheide. $^{20}$ Da die Richtigkeit geisteswissenschaftlicher Forschungsergebnisse häufig, insbesondere im Bereich der Theoriebildung, nicht geprüft oder falsifiziert werden kann, ist es auch schwieriger, über die Güte von Forschungsbeiträgen zu befinden. Deswegen lassen sich auch die Erfolge und Misserfolge von Peer Review in den Geisteswissenschaften nur schwer analysieren und messen.

\subsection{Blind Peer Review}

An der Omnipräsenz von Peer Review im akademischen Alltag gibt es eine Reihe von Kritikpunkten. Eine häufig wiederholte Klage lautet dabei, dass Peer Review den subjektiven Präferenzen der Gutachter unterläge, und damit eine bewusste oder unbewusste Benachteiligung einzelner Wissenschaftler oder Wissenschaftlergruppen einherginge. ${ }^{21}$ Eine verbreitete Maßnahme, um diesen Vor-Urteilen entgegenzuwirken, ist die Maskierung der Identitäten durch das Entfernen der Namen von Autorinnen und/oder Gutachterinnen aus den zu beurteilenden Manuskripten.

Es hat sich für diese Praxis noch keine einheitliche Terminologie durchgesetzt; ich verwende hier die Begriffe von Ann Weller, die Anonymous Peer Re-

$16 \mathrm{Zu}$ den »Dangers of Studying Journal Practices« vgl. auch Chubin / Hackett: Peerless Science, S. 96-98.

17 Shatz: Peer Review.

18 Hall: Academic Self.

19 Vgl. Fitzpatrick: Peer-to-Peer Review, S. 163.

20 Ebd., S. 163.

21 Vgl. z. B. Smith: Peer Review, S. 180; Weller: Editorial Peer Review, S. 207-240. 
view, Single Blind Peer Review und Double Blind Peer Review unterscheidet. ${ }^{22}$ Für das Verfahren, bei dem allen Beteiligten alle Namen bekannt sind, gibt es keinen gängigen Begriff. Anonymous Peer Review bedeutet, dass den Autorinnen nicht mitgeteilt wird, wer ihre Texte begutachtet. Anonym bleibt hier nur der Gutachter. Dies ist die älteste und üblichste Variante der Anonymisierung. ${ }^{23}$ Single Blind Peer Review meint, dass die Gutachterinnen die Namen der Autoren nicht kennen, den Autoren aber der Name der Gutachterinnen mitgeteilt wird. Diese Praxis ist eher selten. Double Blind Peer Review schließlich bedeutet, dass weder die Gutachter die Namen der Autorinnen kennen noch die Autoren die Namen der Gutachterinnen, hier bleiben also beide Seiten einander anonym. Gelegentlich wird ein weiteres Verfahren unterschieden, bei dem auch die Herausgeber den Namen der Autorin nicht kennen. ${ }^{24}$ Für meine Fragestellung nach der Rolle der Namen von wissenschaftlichen Autoren sind sowohl Single als auch Double Blind Review relevant. Da Double Blind Review in der Publikationspraxis wesentlich häufiger vorkommt, werde ich mich im Folgenden vor allem auf diesen Prozess beziehen. Als Oberbegriff für Single und Double Blind Review verwende ich Blind Review für alle Praktiken, bei denen der Name des Autors anonymisiert wird. ${ }^{25}$

Eine zusätzliche Unterscheidung kann zwischen Closed und Open Review gemacht werden. Closed Review ist dabei ein Oberbegriff für alle ReviewVerfahren, die unter Ausschluss der Öffentlichkeit zwischen Herausgeberinnen, Gutachterinnen, Autorinnen und gegebenenfalls Redakteurinnen ablaufen, unabhängig davon, welche Anonymisierungen vorgenommen werden. Dieser Umstand trifft auf die meisten der hier diskutierten Beispiele zu. Open Review hingegen meint eine Reihe von Praktiken, bei denen auf solche Ausschlüsse verzichtet wird und Texte zum Beispiel im Internet frei für Kommentare zugänglich sind. ${ }^{26}$ Sie sollen aber hier nicht Gegenstand der Untersuchung sein. ${ }^{27}$

22 Da die Forschungsliteratur zum Thema zum überwiegenden Teil auf Englisch erschienen ist, benutze ich die englischen Termini. Es existieren deutschsprachige Entsprechungen, die allerdings auch in der deutschsprachigen Forschungsliteratur nicht konsequent verwendet werden. Gerhard Fröhlich spricht z. B. von >(Einfach-)Blindverfahren $\triangleleft$ und $>$ Doppelblindverfahren` (Fröhlich: Anonyme Kritik, S. 39).

23 Vgl. Weller: Editorial Peer Review, S. 211.

24 Fröhlich nennt dies >Dreifachblindverfahren` (Fröhlich: Anonyme Kritik, S. 39).

25 Ich widme mich im Rahmen dieses Aufsatzes nur den Folgen, die eine Anonymisierung von Autornamen als Urhebern von zu veröffentlichenden Artikeln mit sich bringt. Ein Großteil der folgenden Ausführungen kann aber auch auf die Anonymisierung der Namen der Gutachter übertragen werden, die in ihrer Tätigkeit als Autoren von Gutachten ähnlichen Strukturen unterliegen.

26 Vgl. hier zum Beispiel das Projekt MediaCommons Press. 
Für den Zeitraum bis 1999 lässt sich anhand der Meta-Studien von Weller feststellen, dass der Trend in vielen Disziplinen zum Double Blind Peer Review geht. ${ }^{28}$ Sie weist außerdem darauf hin, dass in den Sozial- und Geisteswissenschaften die Praxis des Double Blind Peer Review weiter verbreitet ist als in den naturwissenschaftlichen Fächern. ${ }^{29}$ Weller bezieht sich dabei auf eine Studie von 1984, aber auch in der jüngeren Vergangenheit scheint es sich zu bestätigen: Uwe Thomas Müller untersuchte 3.084 internationale Open-AccessJournals und stellte auch hier fest, dass Double Blind Peer Review in den Geistesund Sozialwissenschaften häufiger praktiziert wird als in Natur- und Ingenieurswissenschaften. ${ }^{30} \mathrm{Zu}$ den Unterschieden zwischen den humanities und den sciences in Bezug auf Peer Review liegen bislang aber kaum Untersuchungen vor.

Bemerkenswert an dieser Entwicklung ist Folgendes: Auch die Einführung des Double Blind Peer Review - als Verbesserung des Vorurteils-anfälligen nicht-anonymisierten Peer Review - geschieht vor allem im Glauben daran, dass dieses Verfahren besser sei als eine Begutachtung, bei der den Gutachtern der Name der Verfasser bekannt ist. Seine Effektivität ist bislang nicht bewiesen. Weller analysiert eine Reihe der bis 1999 verfügbaren Studien, die die Auswirkungen von Anonymisierung auf Gutachterentscheidungen untersucht haben. Sie kommt unter anderem zu dem Schluss, dass in mehr als einem Drittel der Fälle die Gutachter ohnehin treffsicher raten können, von wem ein Artikel geschrieben ist. ${ }^{31}$ Nach der vergleichenden Analyse von sieben verschiedenen Studien zur Effektivität von Blind Peer Review-Prozessen fasst Weller zusammen: »The data are not overly helpful but do show a weak support for a blindreview system «. ${ }^{32}$ Ähnlich wie bei der Analyse von Peer Review im Allgemeinen

27 Für eine Diskussion von Open Review siehe Fitzpatrick: Peer-to-Peer Review, bes. S. 166168. Für eine deutliche Position gegen jede Form von Closed Review siehe Moran: Silencing Scientists. Moran identifiziert Misstrauen und Geheimniskrämerei ('secrecy`) als zentrale Komponenten von Closed Review und betrachtet diese als forschungsfeindlich. Morans Untersuchung argumentiert zwar stellenweise eher normativ als sachlich begründet, liefert aber eine Reihe interessanter Aspekte zum Thema, da seine Beispiele sowohl den Geistes- als auch den Naturwissenschaften entstammen; Moran selbst ist Kunsthistoriker.

28 Vgl. Weller: Editorial Peer Review, S. 238 und Snodgrass: Reviewing, S. 17. Ausnahmen sind u. a. im Bereich der sog. `Big Scienceく zu verzeichnen, weil hier z. B. »die verwendeten Großapparaturen bzw. Verfahren die Forschergruppen hinreichend identifizieren« (Fröhlich: Anonyme Kritik, S. 37).

29 Vgl. Weller: Editorial Peer Review, S. 214.

30 Vgl. Müller: Peer-Review-Verfahren, S. 171.

31 Vgl. Weller: Editorial Peer Review, S. 216.

32 Weller: Editorial Peer Review, S. 239. 
sehen sich empirische Studien zur Effektivität von Blind Peer Review mit dem Problem konfrontiert, dass die intendierten Effekte von Blind Review nicht klar formuliert sind.

Diese Studien beschäftigen sich zum Beispiel mit der Qualität der angenommenen und abgelehnten Beiträge, mit der Qualität der Gutachten selbst oder mit der Zufriedenheit der Autorinnen. Außerdem nehmen sie in den Blick, inwiefern Blind Peer Review sfairer ist als nicht-anonymisierte Verfahren. Hier scheint sich abzuzeichnen, dass Blind Peer Review einige Gruppen schützt, zum Beispiel Frauen, junge Wissenschaftler oder Wissenschaftler von weniger renommierten Institutionen. ${ }^{33}$ Aber auch hier gibt es noch Unklarheiten und widersprüchliche Studien. Es ist zum Beispiel zu vermuten, dass gerade renommierte Wissenschaftler auch nach der Anonymisierung leichter erkannt werden und der Effekt der größeren Fairness dadurch nicht zustande kommt. ${ }^{34}$

Zusammengefasst: Blind Peer Review, also die Anonymisierung eines wissenschaftlichen Textes vor seiner Evaluation, wird in zunehmendem Maße in weiten Teilen der Wissenschaft eingesetzt. Gleichzeitig ist nicht klar formuliert, $\mathrm{zu}$ welchen Zwecken dies geschieht. Die angenommenen und unterstellten möglichen Zwecke, wie Fairness oder Verbesserung der Qualität, lassen sich zudem empirisch nur schwer nachweisen.

Was die qualitativen und quantitativen Studien zur Effektivität des Blind Review Prozesses vollziehen, ist eine Überprüfung der möglichen intendierten Effekte dieses Prozesses. Sie verbleiben dabei auf wissenschaftssoziologischem Boden und untersuchen das Handeln der Teilnehmerinnen an diesem Prozess durch empirische Studien. Ich möchte nun die Perspektive verändern, und versuchen, das Phänomen Blind Peer Review stärker theoretisch zu modellieren. Wenn man die Strukturen in den Blick nimmt, in denen Blind Peer Review vollzogen wird, werden womöglich Eigenschaften des Blind Peer Review sichtbar, die über das wissenschaftliche Alltagshandeln hinausgehen. Dabei möchte ich möglichst stark von den tatsächlichen Prozessen abstrahieren und auf die Unterstellung von Handlungsmotiven der einzelnen Akteure verzichten.

33 Vgl. für eine Übersicht über verschiedene Studien: Weller: Editorial Peer Review, bes. Kapitel 7: Reviewers and their Biases, S. 207-240. Vgl. außerdem z. B. Justice / Cho u. a.: Masking Author, oder Budden / Tregenza u. a.: Female authors.

34 Vgl. Hill / Provost: Double-Blind Review?, S. 179: „One main motivation for double-blind review is to eliminate bias in favor of well-known authors. However, identification accuracy for authors with substantial publication history is even better (60\% accuracy for the top-10\% most prolific authors, $85 \%$ for authors with 100 or more prior papers)«. Die Aussagen beziehen sich auf eine Studie zur automatischen Identifizierung von Autoren. 
Ich werde deswegen nun die eben beschriebenen Prozesse des Blind Peer Review im Rahmen der Luhmann'schen Systemtheorie reformulieren. Ziel ist es dabei, Effekte des Blind Peer Review aufzuzeigen, die in den Studien zur Wirksamkeit dieses Prozesses nicht berücksichtigt worden sind. Wie zu zeigen sein wird, ist das Prinzip der Anonymisierung wissenschaftlicher Autorschaft aus dieser Perspektive nicht nur ein Prozess zur Verbesserung der Qualität wissenschaftlicher Publikationen oder der wissenschaftlichen Fairness, sondern transportiert gleichzeitig Aussagen über ein bestimmtes wissenschaftliches Selbstverständnis.

\section{Blind Peer Review im Wissenschaftssystem}

Luhmann identifiziert als den Code des Wissenschaftssystems mit Bezug auf das generalisierte Kommunikationsmedium >Wahrheit` die Unterscheidung wahr/unwahr. ${ }^{35}$ Wissenschaft verteilt mithilfe ihrer systemeigenen Strukturen und Prozesse die Zuordnung dieser Unterscheidung. Gleichzeitig bestimmt Luhmann als Nebencode der Wissenschaft die >Reputation<. Diese wird, wiederum aufgrund systemeigener Strukturen und Prozesse, als ein Mehr oder Weniger an Reputation innerhalb des Sozialsystems Wissenschaft verteilt. Reputation dient der Orientierung, Selektion und damit der Reduktion von Komplexität. Beide Codes, Wahrheit und Reputation, spielen für das Prinzip der Anonymisierung wissenschaftlicher Arbeiten zur Qualitätssicherung eine Rolle.

\subsection{Reputation}

Der intuitiv leichter $\mathrm{zu}$ erschließende dieser beiden Codes ist der Nebencode >Reputation`. Reputation spiegelt auf leicht ersichtliche Weise die Tatsache, dass Wissenschaft ein soziales System ist, also auf Kommunikation beruht, oder, präziser ausgedrückt, durch Kommunikation seine Autopoiesis betreibt. Der Code >Reputation ‘ ist bei Luhmann explizit an den Namen des Wissenschaftlers gekoppelt: »Reputation wird an Eigennamen verliehen, also an semantische Artefakte mit eindeutiger, rigider Referenz. Die Namen selbst haben,

35 Ich verzichte an dieser Stelle auf eine ausführliche Einführung in die Terminologie Luhmanns, da die in Frage stehenden Strukturen sich auch mit einem basalen Verständnis der Systemtheorie nachvollziehen lassen sollten. Ich beziehe mich im Folgenden mit allen Begriffsverwendungen und Zusammenfassungen auf Luhmann: Wissenschaft der Gesellschaft. 
eben wegen dieser Rigidität, keine eigene wissenschaftliche Bedeutung «. ${ }^{36}$ Reputation wird nicht grundlos an solche Namen verteilt: »Kein Reputationssystem könnte sich halten, wenn die Reputation willkürlich oder in vielen Fällen ganz unverdient erworben werden könnte«. ${ }^{37}$ Er schränkt aber zusätzlich ein, dass die Vergabe von Reputation notwendigerweise Übertreibungen und Untertreibungen einschließt: »Wer oder was Reputation hat, hat mehr Reputation, als er, sie oder es verdienen «. ${ }^{38}$ Die Verteilung von Reputation, so Luhmann, beruht zum Teil auf Reputation selbst, ist also ein "selbstreferentielle[r] Vorgang der Kondensierung von Aufmerksamkeit «. ${ }^{39}$ Die Bündelung von Aufmerksamkeit unter Bedingungen der Zeitknappheit ist es, was Reputation leistet: Bei einer Überfülle von Möglichkeiten - also aus einer Fülle von potenziell wahren wissenschaftlichen Aussagen - dient Reputation als Vor-Selektor dessen, was zur Kenntnis genommen werden muss. Reputation ist an Namen gekoppelt, Verzicht auf Namen bedeutet also Verzicht auf Reputation. Sie, die Reputation, dient auf diese Weise der Reduktion von Komplexität.

Der Nebencode der Reputation erleichtert also die Bewertung von Aussagen innerhalb des Systems Wissenschaft. Gleichzeitig funktioniert der Code >Reputation` aber gegenläufig zu bestimmten sozialen Normen des Wissenschaftssystems: »Der Reputationsmechanismus widerspricht der Norm kollegialer Gleichheit «. ${ }^{40}$ Das Prinzip der kollegialen Gleichheit nennt Luhmann auch die »Fiktion der Gleichheit der Forscher «, ${ }^{41}$ welche die Normen im wissenschaftlich-sozialen Umgang maßgeblich bestimmt. Sie ist, wie ich gleich zeigen werde, unmittelbar an den Begriff der >Wahrheit ' gebunden.

Zusammengefasst: Reputation ist der Nebencode der Wissenschaft. Sie dient der Reduktion von Komplexität. Sie widerspricht aber den sozialen Normen desjenigen Systems, zu dessen Selbsterhaltung sie gleichzeitig beiträgt.

Da die Reputation an den Namen der Wissenschaftlerin gebunden ist, kann in einem einfachen Schritt bestimmt werden, was die Anonymisierung wissenschaftlicher Texte innerhalb des Sozialsystems Wissenschaft bedeutet: Ohne Namen gibt es keine Reputation. Ohne die Möglichkeit der Referenz auf Reputation ist >Wahrheit`, also die Entscheidung wahr oder unwahr, alleiniger Code der Wissenschaft. Ohne Reputation fehlt eine Selektionsorientierung, ein Mechanismus zur Reduktion von Komplexität entfällt und das System erscheint

36 Luhmann: Wissenschaft der Gesellschaft, S. 246.

37 Ebd., S. 251.

38 Ebd., S. 247.

39 Ebd., S. 251.

40 Ebd., S. 354.

41 Ebd., S. 324. 
dementsprechend komplexer. Ohne Reputation kann zudem die Fiktion der Gleichheit der Forscher intakt bleiben, da es keinen Maßstab mehr gibt, mit dem Unterschiede festgestellt werden könnten. Alle drei Punkte sind direkt an das generalisierte Kommunikationsmedium >Wahrheit wahr/unwahr gekoppelt.

\subsection{Wahrheit}

Die Suspension des Nebencodes Reputation durch Anonymisierung von Autorschaft führt also notwendigerweise zu einer Verstärkung der Bedeutung von >Wahrheit`. Ich möchte mich deswegen nun in einem zweiten Schritt dem Medium >Wahrheit` und seiner Bedeutung für das soziale System Wissenschaft zuwenden.

\subsubsection{Die Konstruktion universalen Wissens}

Luhmanns Systemtheorie ist prinzipiell konstruktivistisch und verneint durch das Prinzip der operationalen Geschlossenheit von Systemen die Möglichkeit eines direkten Zugriffs auf die Umwelt. Systemtheoretisch gedacht gibt es Wahrheit immer nur für einen Beobachter. >Wahrheit` kann also systemtheoretisch beobachtet werden, und zwar als symbolisch generalisiertes Kommunikationsmedium im System Wissenschaft.

Für die systemtheoretische Beobachterin wird die Zuordnung ıwahr durch die Wissenschaft nicht für Sachverhalte verliehen, die auch außerhalb der Wissenschaft mit der Wirklichkeit übereinstimmen: Auch Wissenschaftler haben keinen unmittelbaren Kontakt zur Welt. Auch Wissenschaftlerinnen können nicht beobachten, was >ist`, und dann sagen: »Das ist wahr«. Was Wissenschaft stattdessen leistet, ist ein komplexes System zu entwickeln, mit dessen Hilfe bestimmt wird, was als ıwahr schaft stellt also nicht fest: »Das ist wahr ", sondern schafft zuallererst die Möglichkeit überhaupt festzulegen, was als wahr bestimmt werden kann. Und dies vollzieht die Wissenschaft nicht im Rückgriff auf die Welt, sondern auf sich selbst. "Dies heißt nicht, Umwelt zu leugnen, aber die Form, mit der sich das System zur Umwelt in Beziehung setzt [...] ist eine Eigenleistung des Systems «. ${ }^{42}$ Gleichzeitig bildet das Sozialsystem Wissenschaft Strukturen aus, die es ihr

42 Luhmann: Wissenschaft der Gesellschaft, S. 288. 
ermöglichen, so zu sprechen als würde sie sich auf die Welt beziehen. Denn das System - von innen betrachtet - reflektiert den Konstruktionscharakter von ,Wahrheit nicht notwendigerweise. Dies wird erst aus der Perspektive eines Beobachters der Wissenschaft möglich.

Mit Luhmann lässt sich die Perspektive der Wissenschaft auf diese Sachverhalte wie folgt zusammenfassen: In der modernen Wissenschaft unserer Gesellschaft ist eine der Strategien der Konstruktion von Weltbezug an das Konzept eines erkennenden Subjekts geknüpft. Innerhalb des Systems Wissenschaft nimmt das Subjekt eine privilegierte Position ein, weil es dasjenige ist, das die Wahrheit erkennt, sie entdeckt und mitteilt. Die Gültigkeit dieser wissenschaftlichen Wahrheit ist an Intersubjektivität beziehungsweise kommunikative Nachvollziehbarkeit geknüpft: Wissenschaftlich wahr ist nicht nur das, was ein einzelnes wissenschaftliches Subjekt erkennt, sondern wahr ist nur das, was prinzipiell von jedem erkannt werden könnte. Das heißt, die Wahrheit selbst wird von dem Subjekt erkannt, ist aber nicht mit ihm verbunden, sondern liegt außerhalb des Subjekts, und kann dort von jedem, der weiß, wonach er zu suchen hat, erkannt werden. Wissenschaftliche Wahrheiten sind Objekte, die man entdecken, finden, aufspüren kann. Auf diese Art und Weise funktioniert das System Wissenschaft. Diese Art von Wahrheit produziert und prozessiert das System Wissenschaft.

Wenn die erkenntniskritische Systemtheorie nun dieses System beobachtet, kann sie feststellen: Das Finden von Wahrheit außerhalb des Systems Wissenschaft, das Finden von universalem Wissen also, ist eine Konstruktion des Wissenschaftssystems selbst. Um verstehen zu können, wie diese Konstruktion abläuft, benötigt man die Unterscheidung zwischen Erleben und Handeln: »Von Erleben soll immer dann die Rede sein, wenn die Zustandsänderung eines Systems (=Verhalten) dessen Umwelt zugerechnet wird. Von Handeln soll die Rede sein, wenn die Zustandsänderung eines Systems diesem selbst zugerechnet wird . $^{43}$ Erleben ist also die passive Wahrnehmung eines Ereignisses, dessen Ursache als außerhalb des Subjekts liegend beobachtet wird, während beim Handeln das Subjekt selbst als Ursache attribuiert wird. Der Begriff `Zurechnung « meint in diesem Zusammenhang »immer Beobachtungen eines Beobachters«, denn »ein Beobachter kann als Erleben zurechnen, was ein anderer als Handlung sieht, und umgekehrt «. ${ }^{44}$ Ob eine Zustandsänderung als Handlung oder Erleben zugerechnet wird, liegt also nicht im Wesen der Zustandsänderung

43 Ebd., S. 140-141.

44 Ebd., S. 141. 
selbst begründet, sondern hängt vom Beobachter und dessen Systemzugehörigkeit ab.

Das Prinzip der erfahrbaren, wissbaren Welt, das es der Wissenschaft ermöglicht, universal gültiges Wissen zu entdecken, beruht auf der Konvention, Zustandsänderungen des Systems als `Erleben` zuzurechnen. Das Ereignis wird außerhalb des beobachtenden Systems verortet, in diesem Fall außerhalb der Wissenschaft, und ist deswegen auch für alle anderen wissenschaftlichen Beobachter potenziell erlebbar. Es erfüllt damit das Wahrheitskriterium der modernen Wissenschaft.

\title{
2.2.2 Universales Wissen und Anonymität
}

Im Zusammenhang von Erleben und Handeln bringt Luhmann den Begriff der >Anonymisierung` erstmals ins Spiel:

\begin{abstract}
Auf ihre Funktion hin betrachtet, dient die Zurechnung als Erleben [...] der Anonymisierung der Teilnehmer. Wo Handeln interferiert, muß man wissen, wer handelt und welche Interessen und Motive er einbringt. Das gilt auch, wenn es sich um Handeln zum Zwecke des Wissensgewinns handelt. Das Wissen selbst hat seine Geltung dagegen in einer anonym konstituierten Welt. Es kann als Wissen nur überzeugen, wenn man es für prinzipiell gleichgültig hält, wer es erkennt [...]..$^{45}$
\end{abstract}

Die Bedeutung des wissenschaftlich erkennenden Subjekts, die Zurechnung auf Erleben und die daraus resultierende Indifferenz gegenüber eben jenem Subjekt funktionieren allesamt als Fluchtlinien auf einen Punkt hin: auf den des universal gültigen Wissens. »Die Reduktion auf Erleben und die Anonymisierung der Erlebenden ist Grundlage für die Prätention, Wissen sei universales Wissen, das heißt: für jeden Beobachter erreichbar «. ${ }^{46}$

Nur um Missverständnissen vorzubeugen: Der Begriff `Prätention` zeigt noch einmal an, dass das hier vorgestellte Prinzip die Strukturen des Sozialsystems Wissenschaft bezeichnet, nicht aber Luhmanns eigene Erkenntnistheorie. Diese ist, wie eingangs betont, konstruktivistisch erkenntnisskeptisch. Das Prinzip des universal wissbaren, wissenschaftsunabhängig wahren Wissens ist nur eine Eigenschaft des Wissenschafts-systems. ${ }^{47}$

45 Ebd., S. 143.

46 Ebd., S. 145.

47 Die scheinbare Paradoxie, dass Luhmann selbst als Soziologe innerhalb des Wissenschaftssystems operiert, berührt diesen Umstand nicht. 


\section{Konsequenzen}

Nur mit der Anonymisierung der Teilnehmer durch Zurechnung auf Erleben kann die "Prätention, Wissen sei universales Wissen « ${ }^{48}$ aufrecht erhalten werden. Diese ist damit unmittelbar gekoppelt an die Fiktion der Gleichheit der Forscher: Nur wenn alle Forscher gleich und ihre Namen also gleich gültig sind, kann die Wahrheit als Eigenschaft der Umwelt zugerechnet werden, und ist nicht mehr die Konstruktions-Handlung eines einzelnen, wahrnehmenden Subjekts.

Vor diesem Hintergrund erscheint der Autor in der Wissenschaft als Störfaktor. Der Name des Autors, und mit ihm die Reputation, gefährden den Anspruch, universal erlebbares Wissen zu produzieren beziehungsweise zu entdecken. Der Name des wissenschaftlichen Autors gefährdet die Wissenschaftlichkeit der Wissenschaft nach ihren eigenen Regeln. Der Name des Autors schwächt das Prinzip des universalen Wissens.

Dennoch scheint das gegenwärtige Wissenschaftssystem nicht ohne Namen auskommen zu können. Luhmann hält den Namen des Wissenschaftlers für so maßgeblich im System Wissenschaft, dass er das Prinzip `Nebencode konzipiert, um die >Reputation « ins Spiel bringen zu können. Eine Wissenschaft ohne die Namen von Wissenschaftlern erscheint zwar einerseits das erstrebenswerte Ideal, ist andererseits aber mit ihren sozialen Praktiken nicht zu vereinbaren. Der Rückgriff auf Namen ist der Wissenschaft als Sozialsystem inhärent, aber ihr Konzept der Wahrheit ist damit nur schwer vereinbar.

Das Prinzip der Anonymisierung von wissenschaftlichen Artikeln zum Zwecke ihrer Evaluation scheint deswegen eine deutliche Funktion zu erfüllen: Wissenschaft kann nicht ohne den Namen, kann nicht ohne Reputation funktionieren, aber sie kann eine Art Nische schaffen, in der das Wissen mit Anspruch auf Universalisierbarkeit freien Lauf erhält: Wie würde dieser Text verstanden werden, wenn er keinen Autor hätte? Wie würde dieser Text verstanden werden, wenn niemand für ihn bürgte außer der erlebbaren Welt? Blind Peer Review schafft eine Praxis, in der Wissen als nicht an die Erkenntnis eines spezifischen Subjekts gebunden präsentiert wird und realisiert so die »Fiktion der Gleichheit der Forscher «.

Ich hatte die Frage gestellt, welche Effekte die Anonymisierung von Autorschaft haben könnte, die nicht offen zutage treten und die in den Studien zu Peer Review und Blind Peer Review möglicherweise nicht berücksichtigt worden sind.

48 Luhmann: Wissenschaft der Gesellschaft, S. 145. 
Einer dieser strukturellen Effekte kann jetzt benannt werden. Die Anonymisierung der Teilnehmer an Wissenschaft ist immer auch eine Positionierung zum Prinzip des universalen Wissens: die Bewertung von Wissenschaft soll ausschließlich an erlebbarer Wahrheit orientiert sein.

Das Prinzip der Anonymisierung von wissenschaftlicher Autorschaft kann also als Verstärkung einer bestimmten epistemologischen Position verstanden werden: Anonymisierung installiert das Prinzip des intersubjektiven oder subjektlosen Erkennens in der Wissenschaft. In der Praxis des Blind Peer Review geschieht dies an einer wichtigen Entscheidungsschwelle für wissenschaftliche Sichtbarkeit und Anerkennung, nämlich vor der Publikation wissenschaftlicher Ergebnisse. Blind Peer Review führt bei genauerer Betrachtung vor Augen, dass der Autor, das Subjekt, der Urheber, ein Störfaktor in der Produktion von wissenschaftlicher Wahrheit ist.

Blind Peer Review als Qualitätssicherungsstandard der Wissenschaft ist eine Erfindung des zwanzigsten Jahrhunderts. Nicht erst dieses Jahrhundert ist aber geprägt durch eine große Zahl erkenntniskritischer Unternehmungen, die das Prinzip der universal gültigen Wahrheit theoretisch unterlaufen. Luhmann ist nur ein Beispiel für ein post-ontologisches, konstruktivistisches Modell. Es ist deswegen mehr als fraglich, ob Luhmanns Rekonstruktion des Wissenschaftssystems tatsächlich auf alle Bereiche der gegenwärtigen Wissenschaft anwendbar ist. Unter der Bezeichnung >Wissenschaft ‘ agieren in der heutigen akademischen Landschaft durchaus Teilsysteme, die den Wahrheitsbegriff - wie ja auch Luhmann selbst - kritisch hinterfragen und mit konstruktivistischen Theorien arbeiten. Einige Teile insbesondere der kulturwissenschaftlich orientierten Literaturwissenschaft fallen in diese Kategorie.

Was Luhmann als Teil der dem System verborgenen Eigenschaften von Wissenschaft beobachtet - die Konstruktion von Wahrheit innerhalb des Systems ist auch Teil von Theorien, mit denen diese Wissenschaft operiert. Die Skepsis an universal wissbarem, >wahren Wissen ist kein Privileg einer systemtheoretischen Beobachterin, sondern Teil von Kultur- und Gesellschaftstheorien, mit denen innerhalb des Systems Wissenschaft gedacht und gearbeitet wird. ${ }^{49}$ Auch in diesen Wissenschaftszweigen aber wird Blind Peer Review angewendet, obwohl es an das Prinzip des universal wissbaren Wissens gebunden ist. Diesen Widerspruch kann man nun auf zwei Weisen interpretieren:

1. Das Prinzip des universal wissbaren Wissens kann, bei aller konstruktivistischen Theoriearbeit, nicht aus den Arbeitsweisen der Wissenschaft ver-

49 Vgl. z. B. Clam: Differenz. 
bannt werden. Dass über den Prozess des Blind Peer Review an zentraler Stelle weiterhin unter Bedingungen der erlebbaren Erkenntnis und daraus resultierender Wahrheit operiert wird, zeigt, dass auch konstruktivistischreflektierte Teilsysteme der Wissenschaft sich von diesem Grundsatz nicht oder nur schwer lösen können.

2. Gerade die Tatsache, dass eigens Strukturen geschaffen werden, um das subjektlose Erkennen zu simulieren, zeigt, wie schwach dieses Prinzip in der gegenwärtigen Wissenschaft verankert ist. Wenn das Prinzip des universal wissbaren Wissens ein Grundsatz jeglicher Wissenschaft wäre, bräuchte es keinen eigenen Prozess, um diesen Umstand abzusichern und zu prüfen. Wenn es eine komplizierte Nische braucht, um so zu tun, als wäre Wahrheit ohne Autorschaft zu haben, dann liegt es daran, dass Autorschaft, das erkennende Subjekt und seine jeweiligen Konstruktionsleistungen eigentlich als maßgeblich erkannt worden sind.

Der oben zitierte Glaube an das Prinzip des Blind Peer Review könnte also zwei Effekte für die Wissenschaft haben: Entweder ist Blind Peer Review eine wichtige Bastion der universalen Wahrheit in wissenschaftlichen Erkenntnisprozessen, oder aber es ist, als ausgegliederte Nische, ein Abgesang auf die Rolle, die wahre Wahrheiten für die Wissenschaft haben können. Die Diskussion um die Bedeutung wissenschaftlicher Autorschaft liefert also eine Reihe weiterer zu erforschender Fragen. Insbesondere wird es darum gehen müssen, eine Untersuchung der gängigen Praktiken nicht nur mit empirischen Methoden nachzuvollziehen, sondern mit kritischem Blick auch zugrundeliegende Strukturen offenzulegen. Dazu muss immer auch die Perspektive der Wissenschaftsforschung gehören, mit deren Hilfe die Strukturen der Wissenschaft auf diese selbst angewendet werden können. Erst vor dem Hintergrund einer solchen theoretisch informierten Analyse sollten aufbauende empirische Studien durchgeführt werden. ${ }^{50}$

50 Ich danke den Teilnehmerinnen und Teilnehmern an der Diskussion zu meinem Vortrag für wertvolle Hinweise und den Herausgebern für ihre klugen Ergänzungen zu meinem Text. 


\section{Bibliographie}

Biagioli, Mario: „From Book Censorship to Academic Peer Review«. In: Emergences: Journal for the Study of Media \& Composite Cultures 12 (2002) H. 1, S. 11-45.

Budden, Amber E. / Tom Tregenza / Lonnie W. Aarssen / Julia Koricheva / Roosa Leimu / Christopher J. Lortie: "Double-blind review favours increased representation of female authors«. In: Trends in Ecology and Evolution 23 (2008) H. 1, S. 4-6.

Burnham, John C.: »How Journal Editors Came to Develop and Critique Peer Review Procedures». In: H.F. Mayland / R.E. Sojka (Hrsg.): Research Ethics, Manuscript Review, and Journal Quality. Madison, WI 1992, S. 55-62.

Casadevall, Arturo / Ferric C. Fang: »Is Peer Review Censorship?«. In: Infection and Immunity 77 (2009) H. 4, S. 1273-1274.

Chartier, Roger: "Foucault's Chiasmus. Authorship between Science and Literature in the Seventeenth and Eighteenth Centuries«. In: Mario Biagioli / Peter Galison (Hrsg.): Scientific Authorship. Credit and Intellectual Property in Science. New York / London 2003, S. $13-$ 31.

Chubin, Daryl E. / Edward J. Hackett: Peerless Science. Peer Review and U.S. Science Policy. Albany, NY 1990.

Clam, Jean: Was heißt, sich an Differenz statt an Identität orientieren? Zur De-ontologisierung in Philosophie und Sozialwissenschaft. Konstanz 2002.

Fitzpatrick, Kathleen: »Peer-to-peer Review and the Future of Scholarly Authority«. In: Social Epistemology 24 (2010) H. 3, S. 161-179.

Foucault, Michel: „Was ist ein Autor?«. In: Jannidis, Fotis / Gerhard Lauer / Matías Martínez / Simone Winko (Hrsg.): Texte zur Theorie der Autorschaft. Stuttgart 2000, S. 198-229.

Fröhlich, Gerhard: »Anonyme Kritik. Peer Review auf dem Prüfstand der Wissenschaftsforschung «. In: medizin bibliothek information 3 (2003) H. 2, S. 33-39.

Haack, Susan: »Peer Review and Publication: Lessons for Lawyers«. In: Stetson Law Review 36 (2007) H. 3, S. 789-819.

Hall, Donald: The Academic Self: An Owner's Manual. Columbus 2002.

Hill, Shawndra / Foster Provost: "The Myth of the Double-Blind Review? Author Identification using only Citationsu. In: SIGKDD Explorations 5 (2003) H. 2, S. 179-184.

Jefferson, Tom / Elizabeth Wagner / Frank Davidoff: »Measuring the Quality of Editorial Peer Review«. In: Journal of the American Medical Association 287 (2002), S. 2786-2790.

Jefferson, Tom / Philip Alderson / Elizabeth Wagner / Frank Davidoff: „Effects of Editorial Peer Review. A Systematic Review«. In: Journal of the American Medical Association 287 (2002), S. 2784-2786.

Justice, Amy C. / Mildred K. Cho / Margaret A. Winker / Jesse A. Berlin / Drummond Rennie: "Does Masking Author Identity Improve Peer Review Quality?«. In: Journal of the American Medical Association 280 (1998), S. 240-242.

Luhmann, Niklas: Die Wissenschaft der Gesellschaft. Stuttgart 1992.

MediaCommons Press, http://mediacommons.futureofthebook.org/mcpress/ (Stand: 22.08.2011).

Modern Language Association of America: Report of the MLA task force on evaluating scholarship for tenure and promotion, 2006.

http://www.mla.org/pdf/task_force_tenure_promo.pdf (Stand: 25.08.2011). 
Moran, Gordon: Silencing Scientists and Scholars in Other Fields: Power, Paradigm Controls, Peer Review, and Scholarly Communication. Greenwich, CT 1998.

Müller, Uwe Thomas: Peer-Review-Verfahren zur Qualitätssicherung von Open-Access-

Zeitschriften. Systematische Klassifikation und empirische Untersuchung. Berlin 2008.

Shatz, David: Peer review: A critical inquiry. Lanham, MD 2004.

Smith, Richard: „Peer review: a flawed process at the heart of science and journals«. In: Journal of the Royal Society of Medicine 99 (2006), S. 178-182.

Snodgrass, Richard: „Single- Versus Double-Blind Reviewing: An Analysis of the Literature«. In: SIGMOD Record 35 (2006) H. 3, S. 8-21.

Spier, Ray: »The history of the peer-review process«. In: Trends in Biotechnology 20 (2002) H. 8, S. 357-358.

Weller, Ann: Editorial Peer Review: Its Strengths and Weaknesses. Medford, NJ 2001. 